\title{
H.264 Video transmission over WiMAX and ADSL network
}

\author{
Kadhim Hayyawi Flayyih ${ }^{1}$, Prof. Dr. Nasser N. Khamiss ${ }^{2}$ \\ Master Student, The Informatics Institute for Postgraduate Studies, \\ Iraqi Commission for Computers and Information, Baghdad, Iraq \\ AL Nahrain University-College of Information Engineering, Baghdad, Iraq ${ }^{2}$
}

\begin{abstract}
A wide range of multimedia services like mobile streaming, video conferencing and broadcasting models are the challenges especially when speaking about streaming a video over low bit rate channel, that requires great compression performance, in addition, network friendly scheme, so H.264 and transcoding technique is proposed as solution for streaming video over constant bitrate channel. ADSL and Wimax network is evolving as a appropriate solution to cover long distance areas. In this project an effort has been made to show the behavior of video streaming over ADSL and wimax Network. In this work, we have studied the different QoS requests of applications such as video conferencing, video on demand. Throughput, packet loss, delay and jitter performances of different applications have been examined over wimax and ASDL. Networks are simulated using OPNET 14.5.
\end{abstract}

Keyword: H.264, WiMAX, Buffer, QoS, OPNET 14.5, ADSL Network

\section{INTRODUCTION}

various applications have appeared in easing education over Internet like video conferencing, virtual laboratory and video on demand[1]. Video streaming over low bit rate channel is critical issue. H.264 extended profiles are adopted to use for video conferencing and other application, where the video is compressed and then a stream of bits are generated that is suitable for the available bit rate channel. WiMAX and ADSL network are a last-mile video's services. This study is to clarify performance of video streaming applications over ADSL and WiMAX, four metrics are used (packet loss, delay, jitter, and throughput). The bandwidth management is still one of the critical issue to be solved. Transcoding is one of the most important techniques to make the bit stream more appropriate with available low bitrate channel. Various techniques, like scheduling, traffic shaping, admission control and resource reservation are used to improve QoS in networks. Once packets come from different kinds of traffic at a route or switch, then scheduling techniques deal with packet in a simple and convenient way. Every router must do specific sort of queuing discipline which holds how packets are kept tentatively and then sent. Generally queuing disciplines are First in First Out(FIFO), Weighted Fair Queuing(WFQ) and Priority Queuing(PQ)[2]. Media suppliers are discovering new and modern applications over IP networks contributing in developing video services such as real-time video streaming Video And On Demand (VoD) [3]. The study will use actual movie video trace that is extracted from JM 19, as input to simulation model. Exactly, the Matrix movie for a 10-minute interval will stream to one ADSL video client and 3 WiMAX video client stations from server on the Internet. The rest of paper is expressed as follows, Section 2 Video overview, section 3 H.264 compression process, section4 video traffic, section 5 wimax overview, section 6 OPNET wimax simulation model, Section 7 wimax configuration, section 8 scheduling, section 9 deal with the experiments and results and section 10 the conclusion of the proposed system.

\section{VIDEO OVERVIEW}

Video (Stored video) transmission over network with two modes: the streaming (video streaming) and download mode. Where, in the streaming mode, it is not important to download whole video content, yet it is being played-out while parts of the content are being gotten and decoded. Also streaming video in real-time, has delay, bandwidth and loss requests[3]. In this work attentions going on video streaming, which discusses real-time of stored video. Where in video streaming, video is controlled as a sequence of images or frames that are streamed to the subscriber and showed at a constant frame rate. Video streaming is naturally loss-tolerant and delay-sensitive. There are various transmission and buffering requests from the internet and the client station video player for streaming real-time video[4]. The video content possibly will be categorized by some parameters involving pixel colour depth, video format, coding scheme and frame inter arrival rate. The major problem when streaming video content are bandwidth limitations and the digitized video produces huge amount of data needed to represent the image content. 
Vol. 5, Issue 8, August 2018

A special and temporal redundancy are used by applying compression standard to reduce the amount of bits associated with the video contents,H.264 /AVC can support better compression and quality using to overcome such problem[5]. Video frame rates range from 10 frames to 30 frames per second. This factor is dangerous if network circumstances can impact the frame rates and which, if left uncompensated, essentially damages the quality of playback video. Raw video is encoding by H.264 standard and then kept in storage. Upon customer's demand, a server retrieves compacted video data from storage and then the application-layer quality of service control scheme adjusts bit-streams according to the network condition and QoS desires. Following the adaptation, the transport protocols encapsulated the compacted bitstreams and transmitted packets to the network[3].

\section{H.264 COMPRESSION PROCESS}

The encoder compression input video sequence that is consists of the group of pictures begin with Intra (I) frame and followed by some of predicted $(\mathrm{P})$ or bi-predicted $(\mathrm{B})$ frames. Each frame is consists of blocks; the encoder a predicts the current block based on formerly decoded data, either from the current image or frame using intra prediction or from other images or frames that have been coded and sent using inter prediction. Prediction subtracts from the current block to produce a residual error, then a residual block is transformed, quantized and lastly encoded to be streamed over the channel, the encoding and decoding process is shown in fig. 1.

- Transcoding: transcoding is decoding and re-encoding operation. The recompression can be completed at a lesser bitrate so as to deal with bandwidth variations. Transcoding is used to reduce frame rate or to get bit stream encoding using different video coding standard such as (H.264 or MPG4)[6]. After that, the compressed bits will encapsulated in packet as a syntax and then send them through the channel.

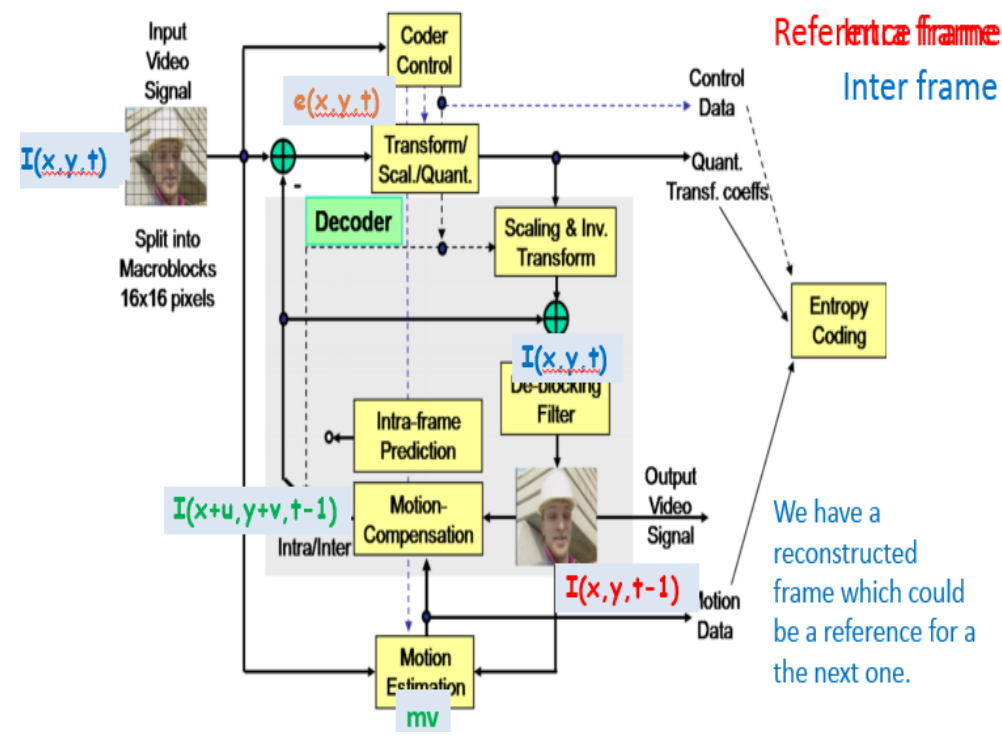

Figure 1 shows the encoder and decoder of H.264 model configuration

This techniques based on rate control and buffering management. Where buffer is existing between video encoder and transmission channel to smooth variation problem. Quality and output rate of the video encoder is organized based on feedback for buffer level. Most effective tools for H.264 are used as controller to control bitrate, video quality and prevent buffer from overflow or underflow. Example of controller, skip frame, Quantization parameter and group of picture, that are corresponding to [5]. Figure 2 show rate control and buffering system.

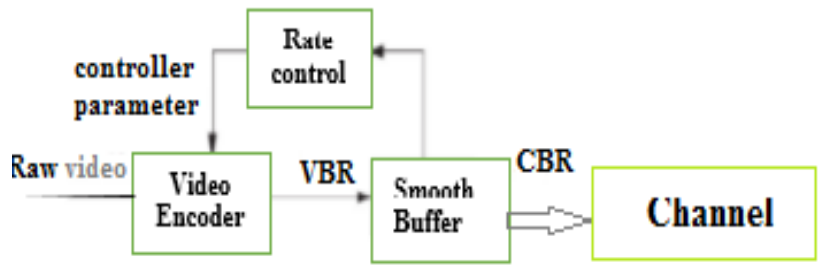

Figure 2 rate control and buffering system 


\title{
International Advanced Research Journal in Science, Engineering and Technology
}

\author{
Vol. 5, Issue 8, August 2018
}

- H.264 syntax layer: H.264 /AVC codec was developed to be good coding, and decoding algorithm for video streaming friendly network, it is separated into (VCL) video coding layer and Network Abstraction Layer (NAL). VCL is a sequence of bits on behalf of the coded video stream which are encapsulated to NAL units former to send or storing VCL data. Every NAL unit comprises a set of data equivalent to coded video data or header facts and a Raw Byte Sequence Payload [7]. NAL units are additional categorized into non-VCL NAL units and VCL NAL units. The VCL NAL units hold coded facts of the picture whereas the non-VCL NAL unit comprises associated extra information like the Picture parameter set (PPS) and the Sequence parameter sets (SPS) [8].The strict transport and encapsulation of NAL units for various transport systems like MPEG-2, H.264 and RTP/IP is shown in figure.3. Streaming services also utilize (UDP) Protocol that supports (BES) best effort service without delay, loss, or bandwidth assurances. UDP is unreliable, connectionless and it does not involve congestion control or flow control compare with Transmission Control Protocol (TCP). However, UDP segments are consequently mapped into unicast IP datagrams for correct addressing and sending to the video clients.

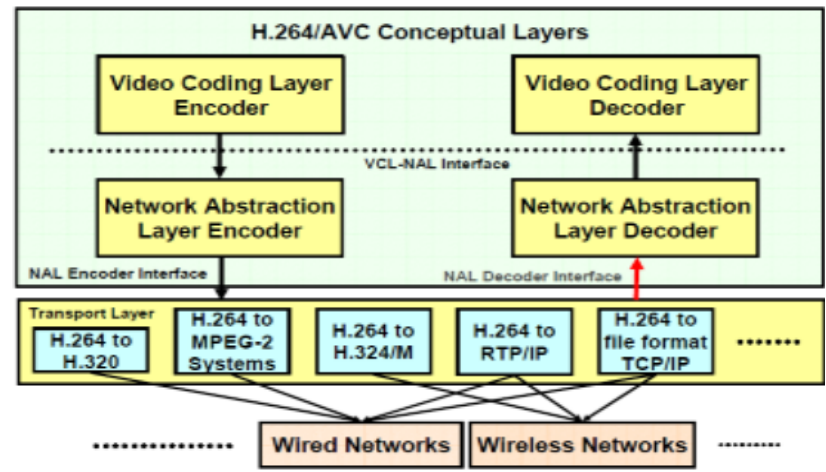

Figure 3 the H.264/AVC Standard in Transport Environment[9].

\section{VIDEO TRAFFIC}

It is a primary issue of this study, where its natural bandwidth exhaustive and delay susceptible properties will pressure the access links further than greatest other kinds of application. Video on demand is compacted video streams are transmitted to the receiver upon request that can be recovered when required. VoD system supports H.264 compression standard, many video sources with different coding parameters were imported to this simulation model. The compression coding parameters are explained with more detail in [5]. Two video traffic are imported into opnet simulator which is coding with H.264 JM 19, as is described inTable1. The first video traffic is real video trace file from a 10 minute H.264 Matrix with a 352x288 resolution and a frame rate of 30 frames per second, which is extract from compression using JM 19 trace file and JM Reference trace parser as show in figure 4.

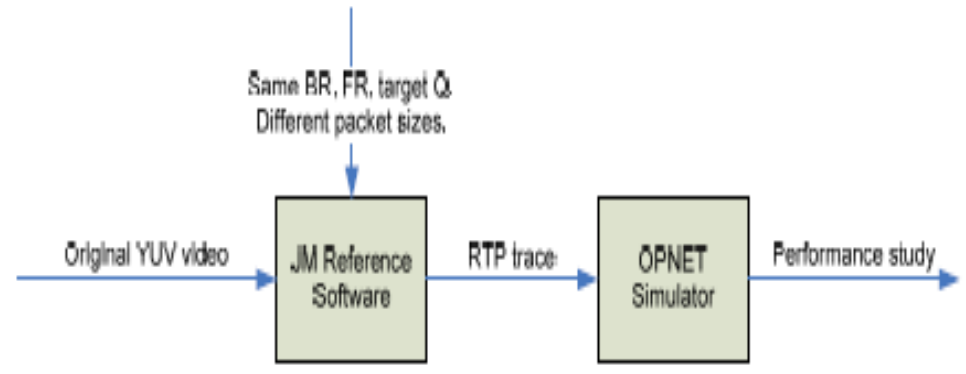

Figure 4: Methodology for video performance study.

Table 1: Characteristics of the video source.

\begin{tabular}{|l|l|l|}
\hline parameter & CIF1 & HD1 \\
\hline Resolution & $352 * 288$ & $1920 * 1080$ \\
\hline Codec & H.264 & H.264 \\
\hline Number of frames & 2999 & 57 \\
\hline Sequence type & I-B-B-P-B-B-P & I-B-B-P-B-B-P \\
\hline GOP & 5 & 12 \\
\hline Frame Rate frames/sec) & 30 & 30 \\
\hline QP & 20 & 30 \\
\hline Number of reference frame & 5 & 2 \\
\hline
\end{tabular}




\title{
International Advanced Research Journal in Science, Engineering and Technology
}

\author{
Vol. 5, Issue 8, August 2018
}

The second video traffic is video trace file from a 10 minute H.264 trace with a $1920 * 1080$ frames resolution and frame rate of 30 frames per second, which is extract using JM 19 trace file. All video traces reflect video frames only and it necessary preprocessing before they could be introduced into the Modeler. Essentially the traces were organized into codec sequence, after that the image or frame sizes were takeout and transformed from bits to bytes. Finally, the video trace files were introduced into the modeler video conferencing frame size configuration by way of a scripted file as show figure 5 .

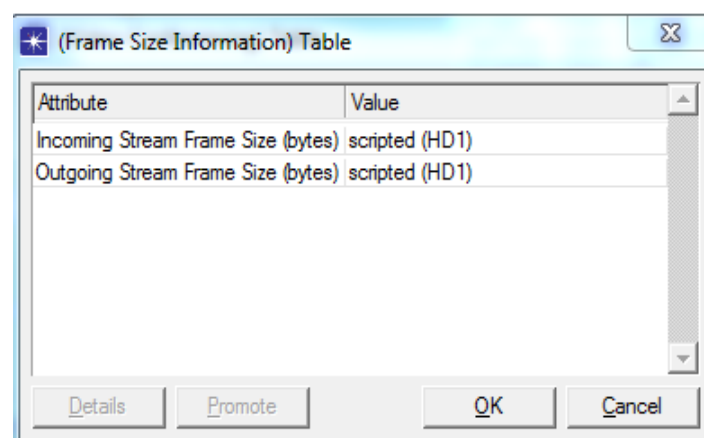

Figure 5(a): frame size.

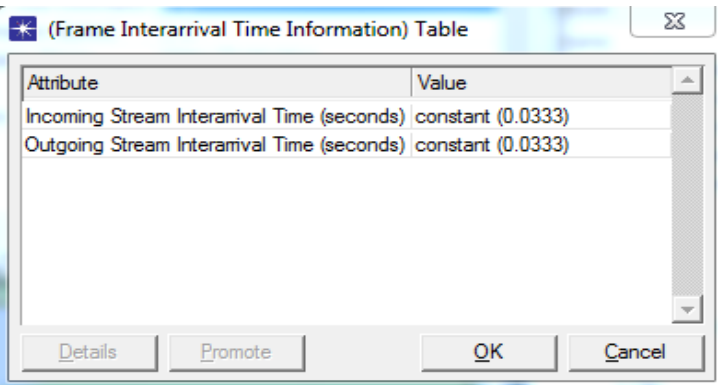

Figure 5 (b): inter arrival time.

\section{WIMAX OVERVIEW}

Wimax has become an attractive solution due to its wireless connectively over a big distance as compared with other advanced wireless technologies, it offers a last mile solution with well coverage to other clients [1]. Wimax based Long Distance (WiLD) network is arising as an appropriate solution for coverage long distance areas (a last mile) compared to other wireless technologies. WiMAX, established on the IEEE 802.16 standard (PHY and MAC layers), is one of many developing technologies of broadband wireless project, it is transmission rate can reach up to $75 \mathrm{Mbps}$ and coverage distance to $50 \mathrm{~km}$. WiMAX enjoys a high transmission speed up to at approximately $100 \mathrm{~km} / \mathrm{hr}[1][3]$. A two versions of WIMAX are existing to handle request for various kinds of access. The IEEE 802.16-2004 WIMAX is the first version. This version provides access in line-of-sight and non-line-of-sight environments and uses Orthogonal Frequency Division Multiplexing (OFDM). In this version, bit rate ranges from $32 \mathrm{Mbps}$ to $134 \mathrm{Mbps}$. IEEE 802.16e WIMAX is second version, which based on 802.16e modification and provides roaming and handoff. This version involves access in non-line-of-sight environments and has a bit rate of up to $15 \mathrm{Mbps}[10]$. The WiMAX network, including Subscriber Station (SS) and Base Station (BS), with two transmission modes of network topology namely, mesh and Point to Multi Point (PMP). WiMAX is capable of achieving QoS, using a bandwidth demand and providing scheme on the subscriber stations, this helping WiMAX base station to keep its existing resources[4].

VI. AN OPNET WIMAX SIMULATION MODEL

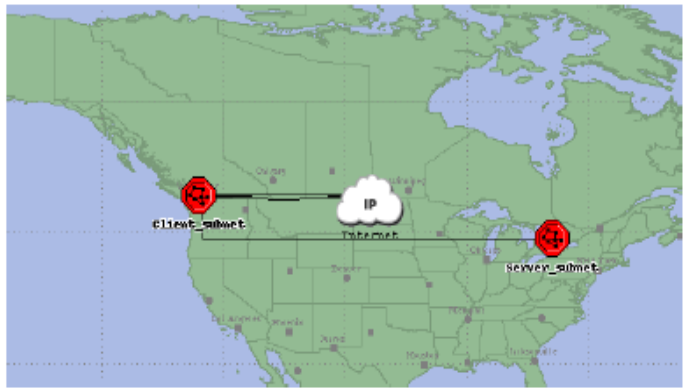

Figure 6: Simulation model network topology. 


\title{
International Advanced Research Journal in Science, Engineering and Technology
}

\author{
Vol. 5, Issue 8, August 2018
}

In this work, OPNET 14.5 is used to improve a simulation model, The OPNET Modeler involved inherent support for both WiMAX and ADSL technologies. A topology for visual network comprising of geographically isolated clients or students and on demand services (instructors) subnets as is shown in figure 6. A server subnet displayed in Figure 7 is found in Toronto, where VoD server able to stream storing video, audio and any other class materials to clients (students) on request. In this subnet the video server is located behind the firewall. The firewall's outdoor interface joins to an access router which is linked to the network via a 45 Mbps wide area network.



Figure 7: Video services subnet

A video streams from server to one or more wimax base station, then deliver to clients within wimax base station in unicast manner as it requested. The video client subnet is located in Vancouver, as show in Figure 8, where, 4 video receiver stations that will demand a VoD services from server. Then the server will response in unicast manner (one copy for each requested client). In this work, the subnet involves three static wireless WiMAX stations are placed at 2 , 4, and $6 \mathrm{~km}$ from the WiMAX base station. The base station is linked to network via a DS3 WAN. Fourth video receiver is an ADSL station placed at $5 \mathrm{~km}$ from the central office. Subnets are connected to the Internet via DS3 WAN circuits. The accurate distance between the two subnets is $3342 \mathrm{~km}$. The LAN and WAN links were designed with interchanging 10 and 20\% utilization loads over 30 minute intervals. Furthermore, the Internet "cloud" was designed with a packet dropped ratio of $0.001 \%$ that outcomes in one packet out of each 100,000 packets is discared in the Internet. The Internet also presents $1 \mathrm{~ms}$ delay as well as the propagation delays showed on the WAN links.

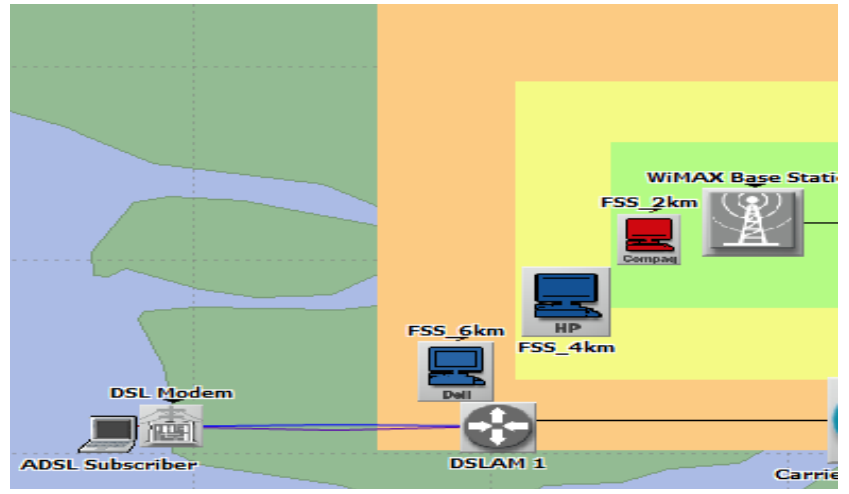

Figure 8: Simulation model of the video client subnet.

The arriving frame rate was formed to invert the content encoding rate. The subsequent unidirectional video stream was mapped to a Type of Service value in order to be later mapped to a WiMAX service flow. The Modeler profile node was designed to invert the recently well-defined applications (validation video conferencing, H.264 movie).

\section{WiMAX CONFIGURATION}

The WiMAX model was designed to provide video traffic on only static station. In WiMAX, a service class gets the QoS requests of service flows, where service flows from the subscriber station to base station are called uplink flows and service flows from the base station to the subscriber station are called downlink. For a specified service class, the main factors are lowest sustainable data rate, that is smallest guaranteed over the air rate, in addition to the MAC scheduler kind. The MAC scheduling permits WiMAX to offer QoS abilities like video services. There are four scheduler kinds:

- $\quad$ BE (best effort)

- $\quad$ rtPS (real time polling service)

- UGS (ungranted service)

- $\quad$ nrtPS (Non real-time polling service) 


\title{
International Advanced Research Journal in Science, Engineering and Technology
}

\author{
Vol. 5, Issue 8, August 2018
}

A free bandwidth resources are gives first to USG then to rtPS and nrtPS, finally to BE. For this project, (BE) scheduling was formed for uplink and the downlink to support video on demand. The scheduling was formed with 640 kbps minimum sustainable traffic rate and 1 Mbps maximum traffic rate, as shown in Figure9. Extra service class was generated for the downlink with BE scheduling and 3.0 Mbps minimum data rate. WiMAX subscriber stations and base station were designed to capture down link and the uplink service flows to a particular types of service setting which was formed through the application node configuration. However, every service flow, uplink and downlink can be formed with particular burst profile. In this project, same burst profile used on both downlink and the uplink service flows for a given WiMAX station that have similar properties to the downlink channel and uplink channel. WiMAX receiver stations were manually formed with more forceful modulation/coding systems with gradually increased distance from the base station.16-QAM1/2 scheme was configured for the 4k and 6k fixed subscriber stations and 16QAM $3 / 4$ scheme was configured for the $2 \mathrm{~km}$ fixed subscriber station (FSS). The $2 \mathrm{~km}$ coding rates and modulation for both downlink and uplink service flows are shown in Figure 10.

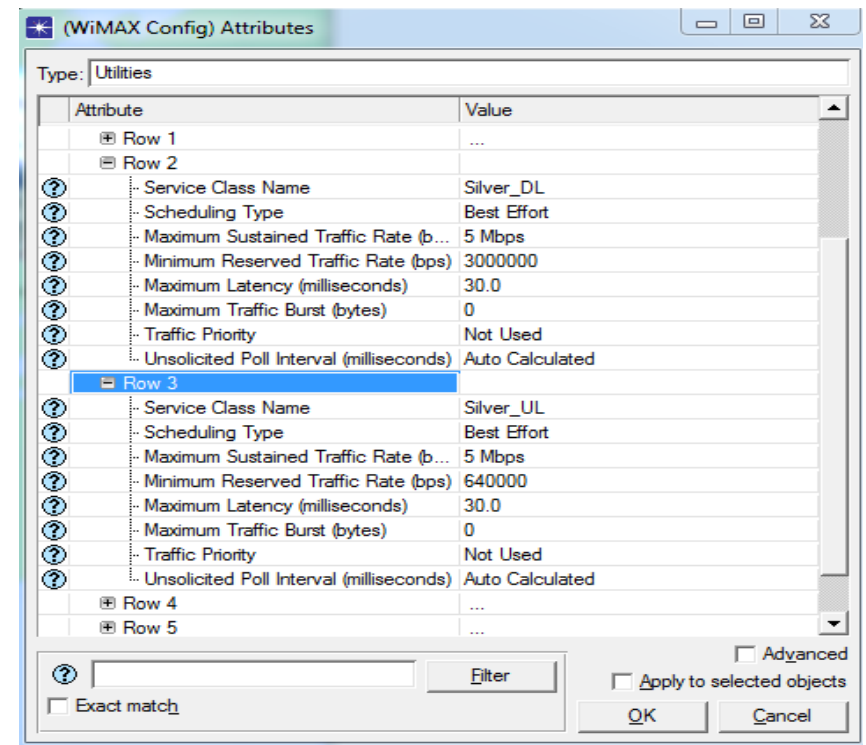

Figure 9: WiMAX service class configuration

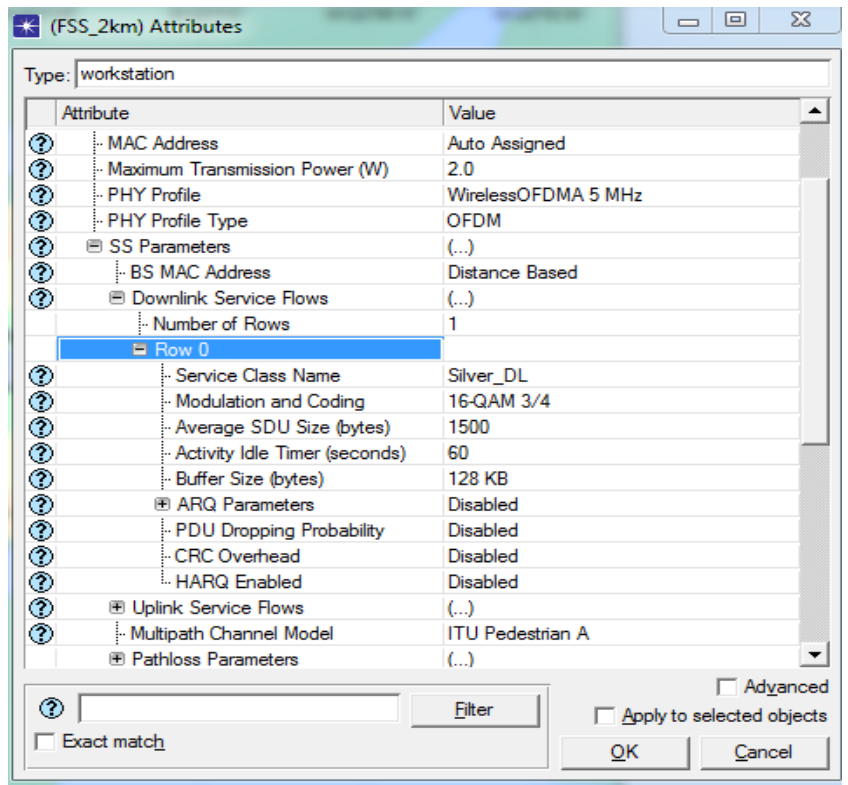

Figure 10: FSS service flow modulation and coding rates.

The Physical (PHY) layer access was formed or designed to exploit OFDM over a $2.5 \mathrm{GHz}$ base frequency using a 5 $\mathrm{MHz}$ network or channel bandwidth that supplies 512 subcarriers assigned. The base station transfer power was designed or configured to $35.8 \mathrm{~dB}$ that matches roughly 3.8 watts with $15 \mathrm{dBi}$ gain antenna as show in figure 11 . On 


\section{International Advanced Research Journal in Science, Engineering and Technology}

Vol. 5, Issue 8, August 2018

the other hand, the user station transfer power was formed to use $33 \mathrm{~dB}$ equal to about 2 watts of transfer power over the $5 \mathrm{MHz}$ network or channel bandwidth, using $14 \mathrm{dBi}$ gain antennas.

ADSL Configuration : The ADSL configuration or design utilized in this model will represent an "enhanced" subscriber package with a $640 \mathrm{kbps}$ uplink channel and 3.0Mbps downlink channel. The distance between the central office and the subscriber was $5 \mathrm{~km}$.

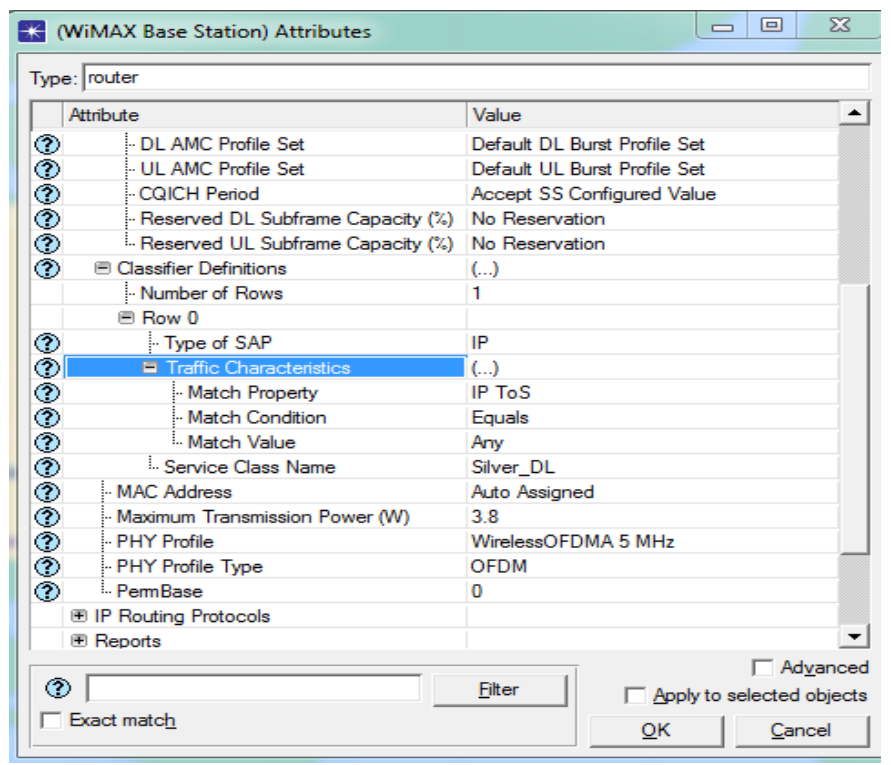

Figure 11: Base Station Parameters

\section{SCHEDULING}

The most generally used queuing disciplines are Weighted Fair Queuing (WFQ), First in First Out(FIFO) and(PQ) Priority Queuing. Various queuing scheduling disciplines in the routers for the IP network can impact on performance of various kinds of Services like video streaming and VoIP, and the exploitation of the internet resources. Figure 12 displays the routers settings of the three queuing scheduling disciplines.

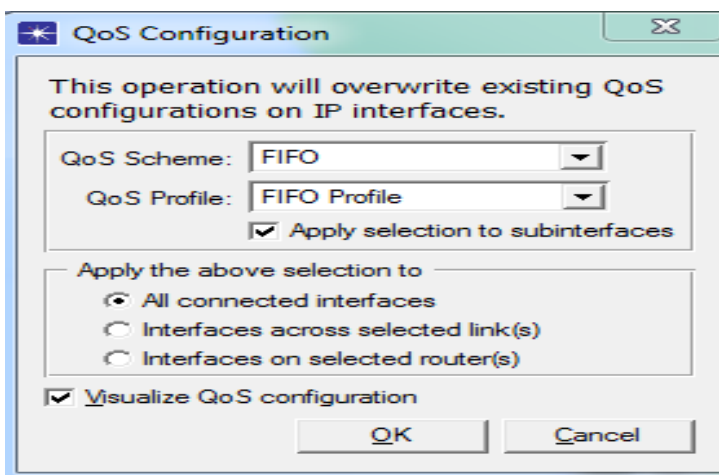

(a)

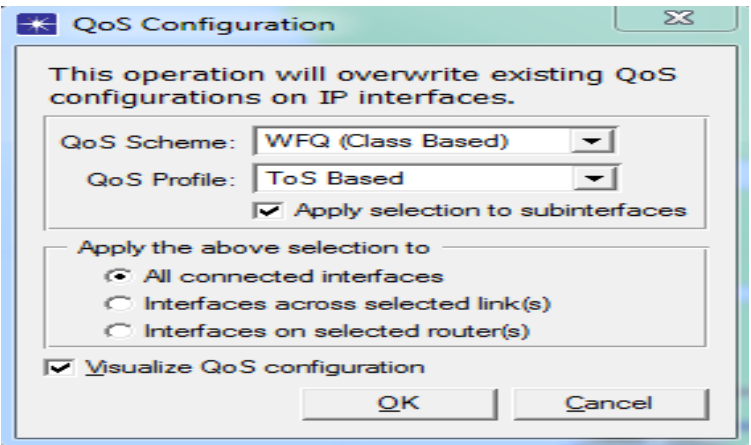

(b) 
Vol. 5, Issue 8, August 2018

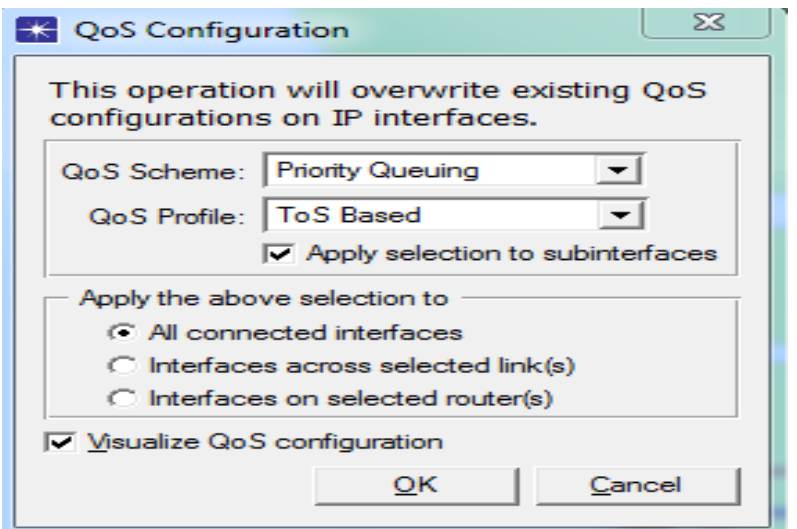

(c)

Fig.12 Router configuration for (a) FIFO (b) WFQ (c) PQ

Further base station buffer size changing was accompanied to discover its effect on discarded packet rate statistic and the video packet loss statistic. Numerous queue sizes ranging from the default value of $64 \mathrm{~KB}$ to $1024 \mathrm{~KB}$ were examined and simulated as show in figure 13

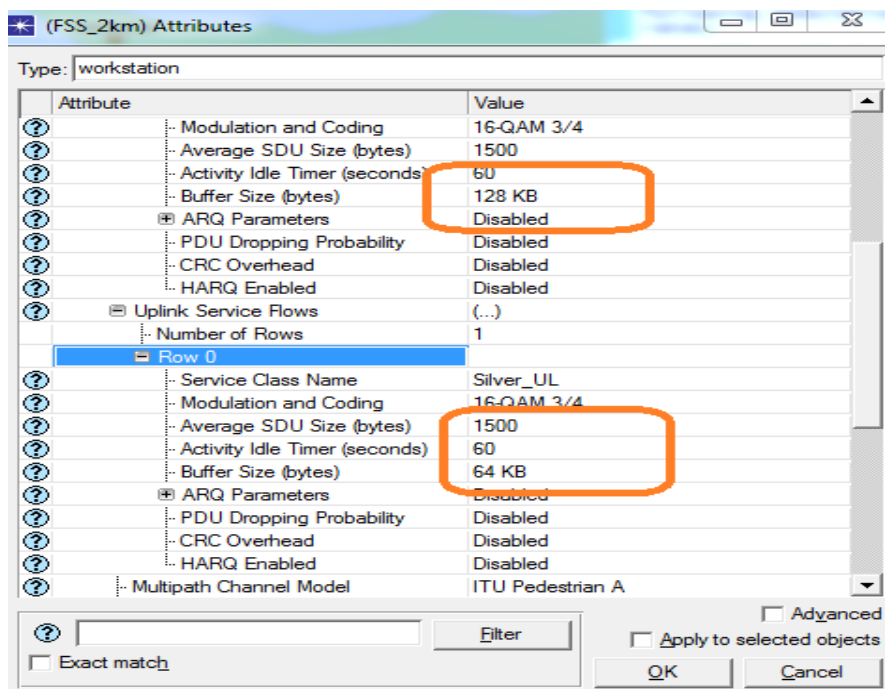

Figure 13: buffer size.

\section{EXPERIMENTS AND RESULTS}

1. WiMAX Link Characteristics: The next PHY layer statistics present perception into the performance of the WiMAX access network. Figure 14 displays the discarded packet rates through the PHY layer for 3 WiMAX stations. The $6 \mathrm{~km}$ WiMAX station (green curve) displays a much greater drop rate than the $2 \mathrm{~km}$ and $4 \mathrm{~km}$ stations over the 10 minute interval.

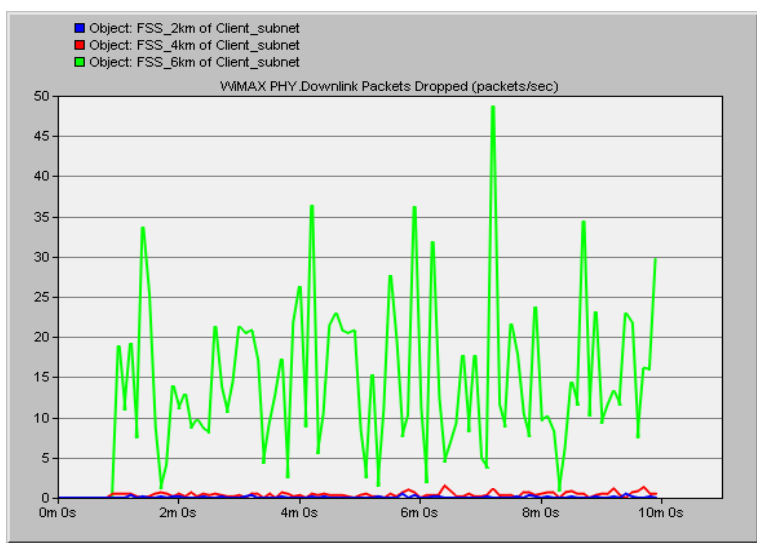

Figure 14: Downlink Dropped pkts/sec 


\section{International Advanced Research Journal in Science, Engineering and Technology}

Vol. 5, Issue 8, August 2018

Figure 15 offers downlink SNR for the three WiMAX stations. See that the $6 \mathrm{~km}$ station shows a downlink SNR that is below the required. This small SNR for the $6 \mathrm{~km}$ station is a major contributor to the great drop rate consequently.

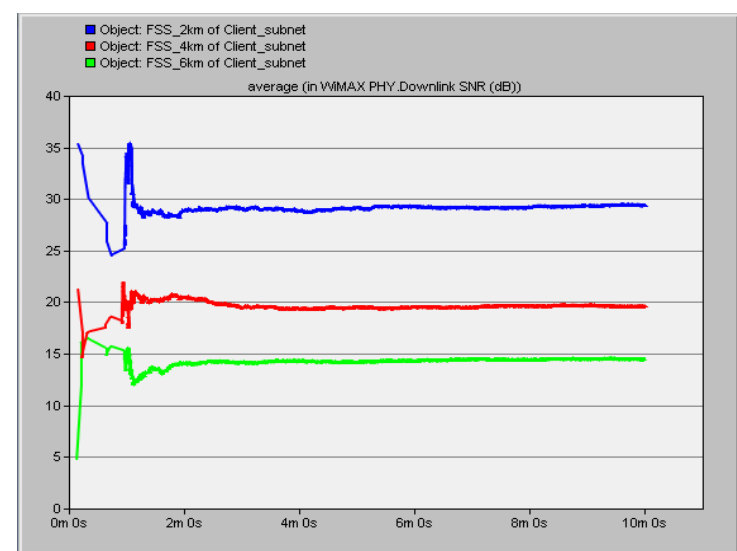

Figure 15: Downlink SNR

A downlink Block Error Rate for $4 \mathrm{~km}$ and the $2 \mathrm{~km}$ WiMAX stations are displayed in Figure 16. The $4 \mathrm{~km}$ station is predictable to invert a greater BLER given that it is double as far from the base station than the $2 \mathrm{~km}$ station. Figure 17 shows a BLER two orders of size greater than the $4 \mathrm{~km}$ WiMAX station.

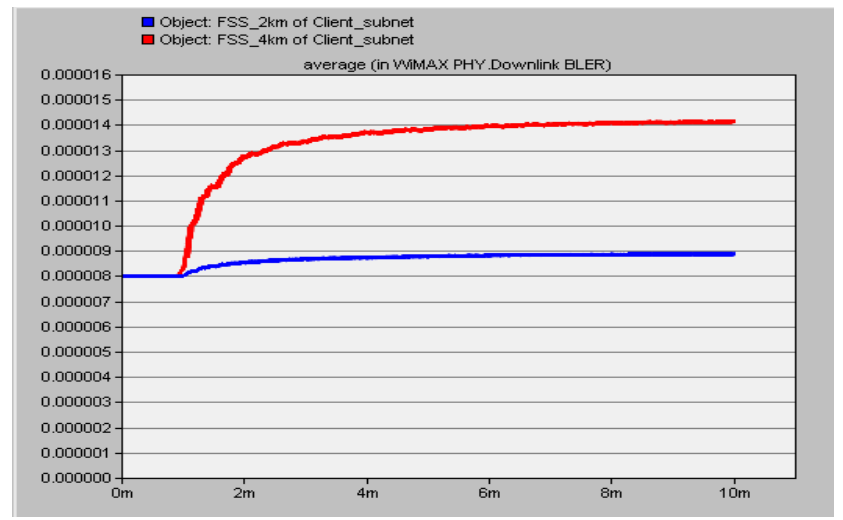

Figure 16: Downlink BLER

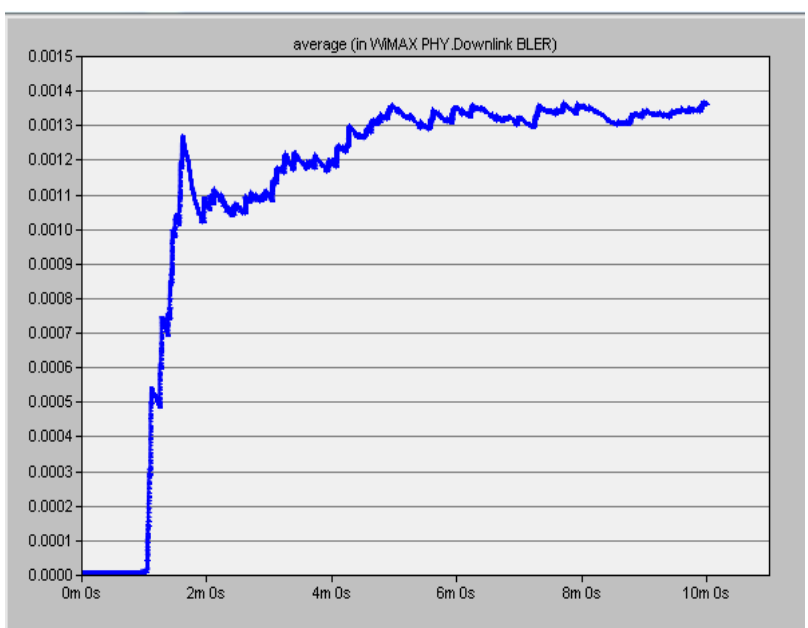

Figure 17: Downlink BLER

2. Video Packet Loss: The succeeding figures display the resulting packet loss detected on all four video clients. OPNET Modeler does not include a video application layer loss measurement. All four curves are be around the 10 minute interval duration. The blue ADSL receiver curve exhibitions a received packet rate arrive to roughly 25 packets/sec, while the WiMAX stations display a variation from the encoding rate with more obvious degradation whenever the subscriber to base station distance rises, as show in figure 18 . 
Vol. 5, Issue 8, August 2018

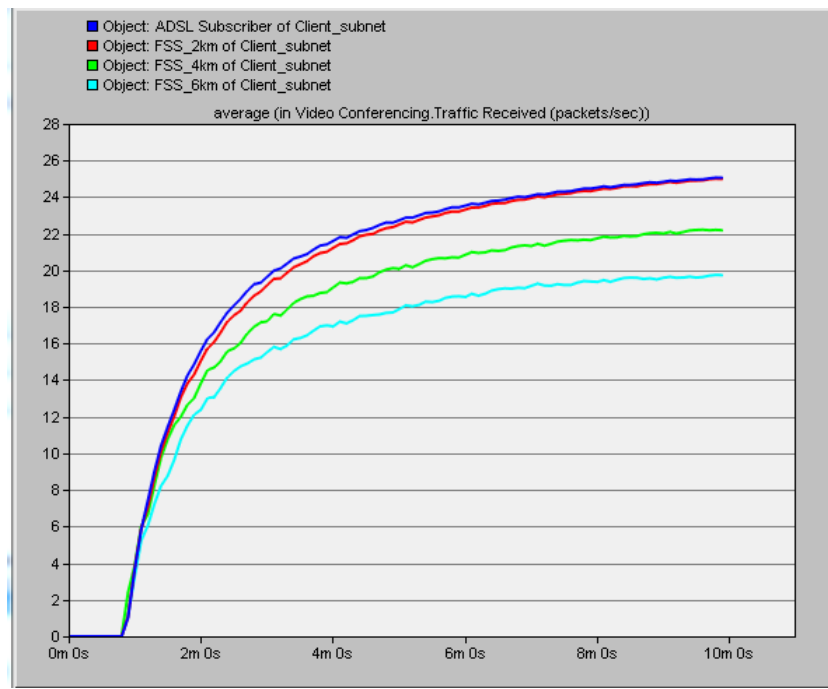

Figure 18: Received video packets/sec

For understanding the reason why the video packet loss on the WiMAX stations is important, more investigation and characterization was essential. Figure 19 maps the $2 \mathrm{~km}$ WiMAX station video packet discard rate along with the MAC

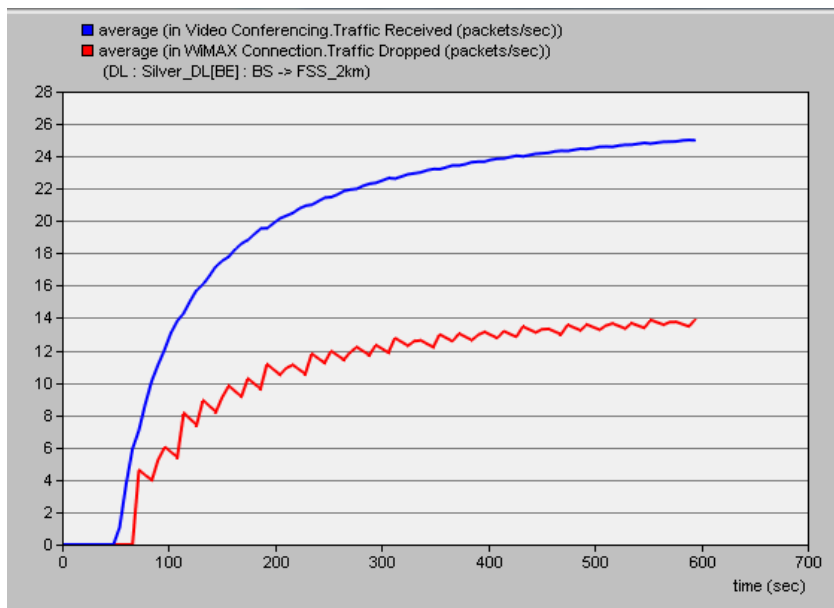

Figure19: Received and dropped pkts/sec

Because of the base station buffer of $128 \mathrm{~KB}$ was being filled, so it is obvious that the MAC layer on the base station is dropping an important number of frames as shown in Figure 20. This performance is largely in part because of the variable sized H.264 video coding frames. Same behavior was perceived with the $4 \mathrm{~km}$ and $6 \mathrm{~km}$ WiMAX stations.



(a) 
Vol. 5, Issue 8, August 2018

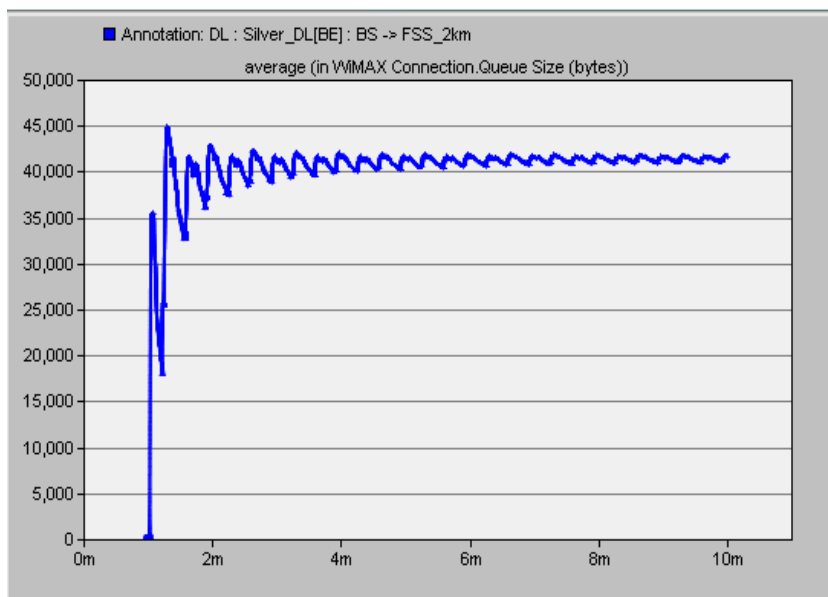

(b)

Figure 20(a), (b): Base station DL queue.

3. Delay: The end-to-end delay computed in the simulation run is displayed in Figure 21. The curves for four video client are averaged through the 10 minute interval. The results show that the ADSL client approaches the best delay of $10 \mathrm{~ms}$ or less. All three WiMAX client station curves closely followed each other while displaying a damping effect that seems to settle between approximately $0.17 \mathrm{~s}$ and $0.13 \mathrm{~s}$ at the end of the movie.

4. Jitter :A video packet jitter computed in the simulation run is explained in Figure 22. The curves for four video client are averaged through the 10 minute interval. The results show that the ADSL client achieved well than ideal value, at approximately $0.004 \mathrm{~s}$. The WiMAX client station curves closely followed each other with a jitter value ranging from $0.036 \mathrm{Mbps}$ to $0.048 \mathrm{Mbps}$, falls within identified metric, which also approached the ideal value.

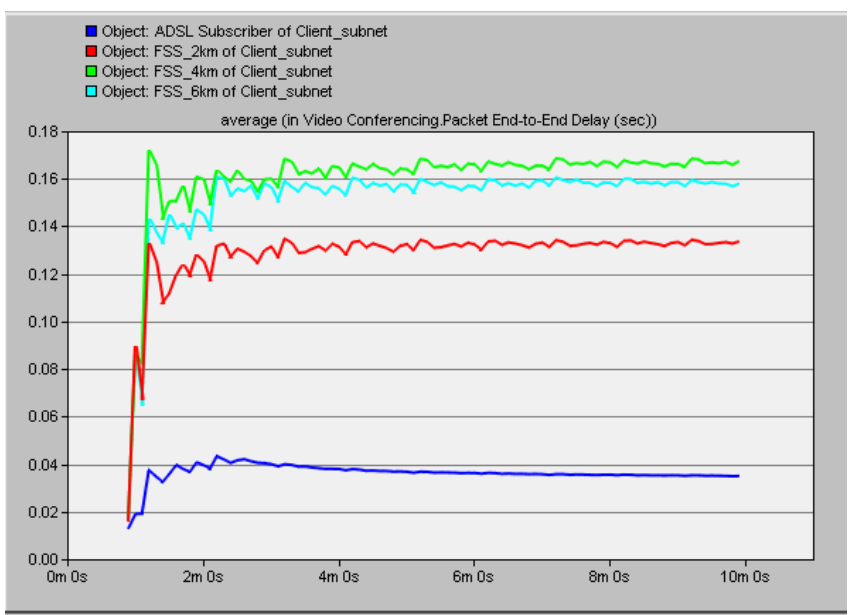

Figure 21: End-to-end packet delay



Figure 22: Video packet jitter 


\section{International Advanced Research Journal in Science, Engineering and Technology}

Vol. 5, Issue 8, August 2018

5. Throughput: The throughput computed in the simulation run is explained in Figure 23. All four client curves followed each other as estimated. The perceived throughputs ranged from $0.40 \mathrm{Mbps}$ to $0.72 \mathrm{Mbps}$ that exists within identified metric and matches to the mean traffic rate for the H.264 content in Table 2.

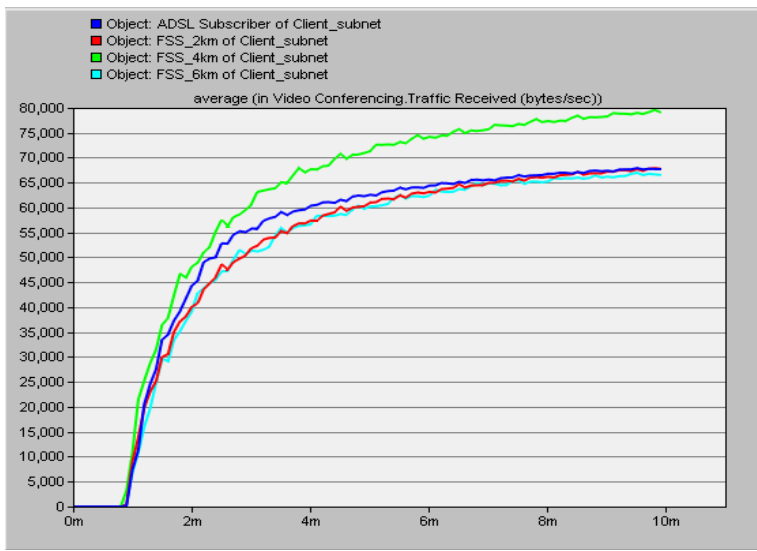

Figure 23: Minimum throughput

6. Video Server :The model was formed and designed to send video content to all 4 video clients using H.264 video content defined in Table 2. Exactly, the movie is coded at a rate of $30 \mathrm{fps}$. VoD server is likely to send out unicast packets for each customer. Figure 24 assured the likely behavior correspondingly.



(a) Video server send bytes/sec

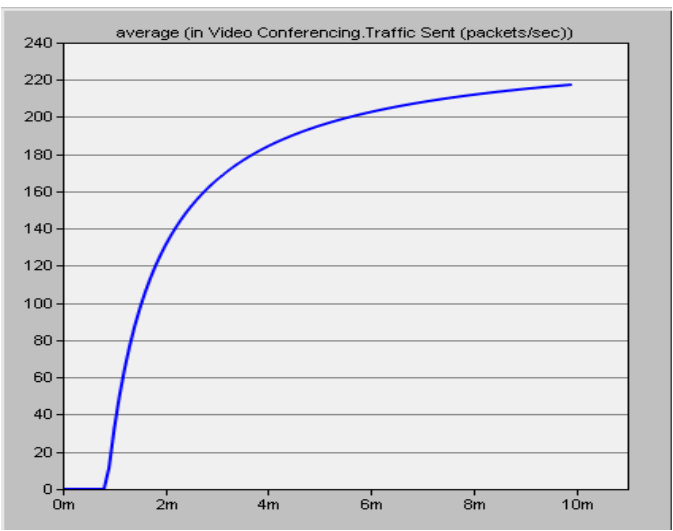

(b)

Figure 24(a), (b): Video server send packets/sec

7. Optimizing buffer size: After take into consideration the behavior described in previously" video packet Loss" section, the buffer size of the base station was further adjusted to explore its effect on the dropped packet rate measurement and eventually the video packet loss measurement. Many queue sizes ranging from the default value from $64 \mathrm{~KB}$ to $1024 \mathrm{~KB}$ were explored and simulated. It was clear that $1024 \mathrm{~KB}$ buffer solution exceeded the requirement resulting in a lower a MAC packet discarded rate of zero. Figure 25 shows much better performance for $2 \mathrm{~km}$ and $4 \mathrm{~km}$ WiMAX stations. While the $6 \mathrm{~km}$ station persistent to show unsatisfactorily great packet loss rates, this was mainly because of resulting SNR that was less the minimum level necessary for the confirmed modulation / coding scheme. 
Vol. 5, Issue 8, August 2018

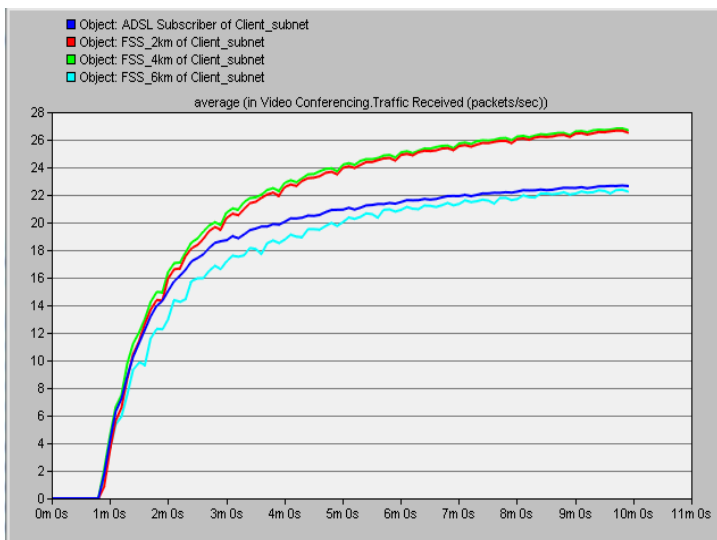

Figure 25: Received Video packets/sec (average)

With additional analysis of the $2 \mathrm{~km}$ WiMAX station, Figure 26 shows the received video packet rate closely follows the original encoding and transfer rate. More, it also sets zero for dropped packet rate which corresponds with Figure 27 which shows the base station connection queue magnitude never arrives its size of $1024 \mathrm{~KB}$.

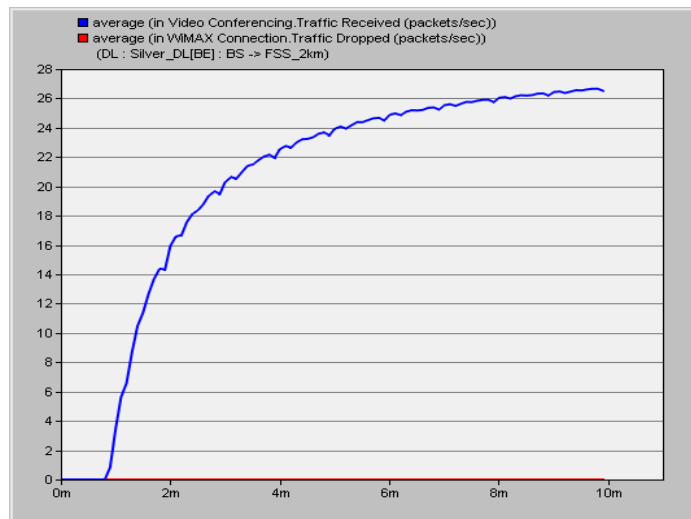

Figure 26: Received/Dropped Video packets/sec

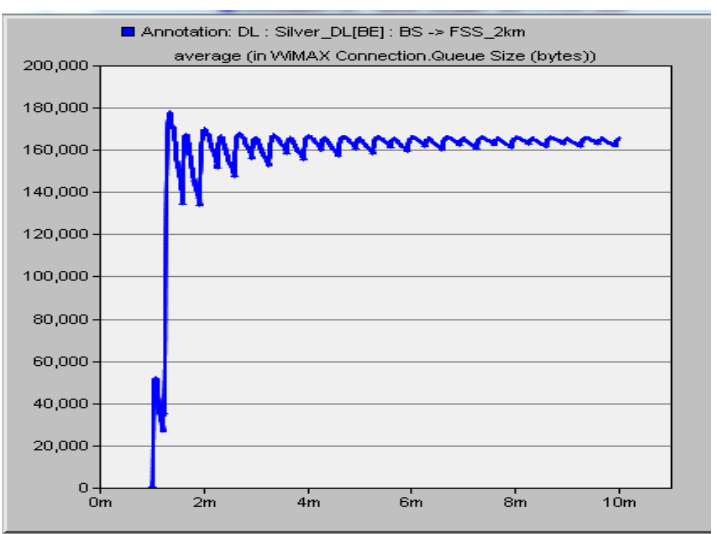

Figure 27: Base station queue size (inst.)

Review of Different Queuing Disciplines in Video Conferencing: Wireless network MANs as required in WiMAX offer wireless broadband access anyplace, anytime and on almost every device. Presenting the different kind of scheduling algorithm, such as PQ, FIFO and WFQ, to compare four types of scheduling services, with their own QoS requirements and the OPNET designer also provided support for the WiMAX network.

Simulated Scenario: The simulation is performed on scenario generated to transfer the Wimax video service using various uplink scheduling algorithm like FIFO, WFQ and Priority Queue, simulation gives the outcomes for packet delay variation, average jitter, packet end-to-end delay and traffic received throughput for video conferencing. Figure 28 displays packet delay fluctuation time for video conferencing, when the traffic or time rises the maximum packet delay alteration time is practiced by the WFQ queuing scheduling discipline. Priority Queue has small delay fluctuation near zero. FIFO have Average jitter value about approximately $4 \mathrm{~s}$ at the end of simulation. 
Vol. 5, Issue 8, August 2018



Figure 28: Packet delay variation

Fig 29 presents the results for end-to-end packet delay (sec). The results show that the PQ algorithm has the smallest delay while the WFQ has high delay value approximately at $2.4 \mathrm{~s}$. Finally, the FIFO have average packet delay fluctuation as well as end-to-end delay.



Figure 29: End to End Delay

Figure30 shows video Conferencing traffic flow received (bytes/sec) for various scheduling algorithms. The upper traffic is received and managed by WFQ because of strict or serve priority and Priority Queue is establish to handle smaller traffic. FIFO has Average traffic that is between the WFQ and Priority Queue.

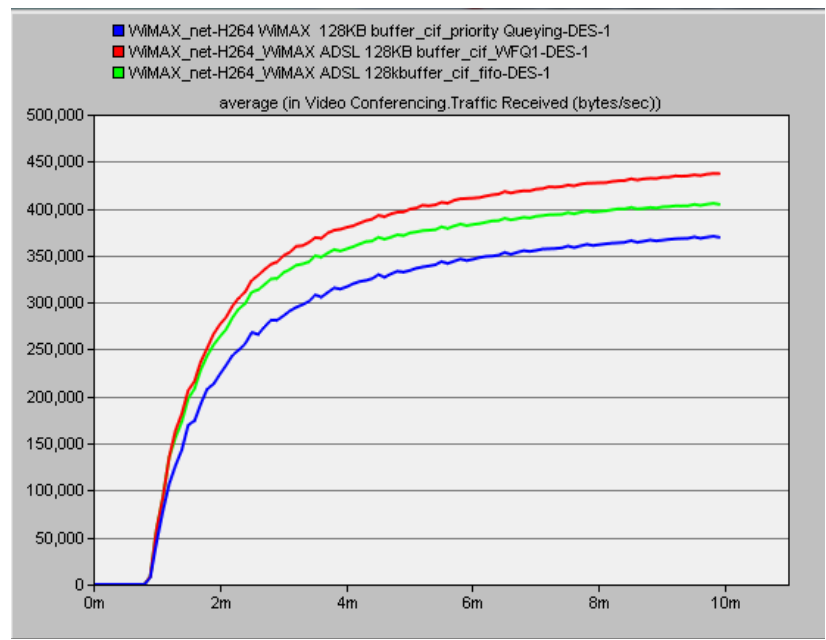

Figure 30: traffic received (bytes) 


\title{
International Advanced Research Journal in Science, Engineering and Technology
}

\author{
Vol. 5, Issue 8, August 2018
}

The maximum of dropped packets rate happens with the FIFO queuing scheduling. The PQ is lesser and tracked by WFQ as is shown in figure 31 .

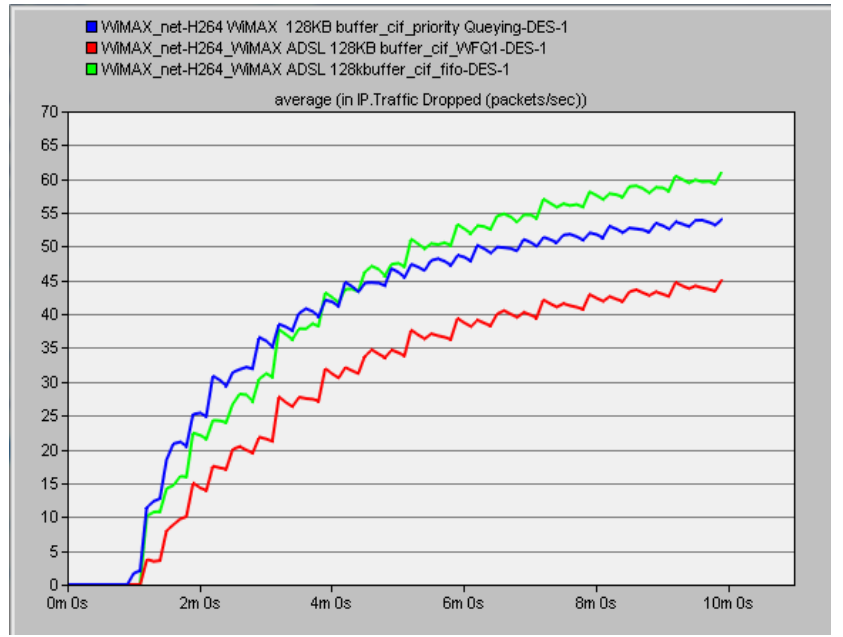

Figure 31: IP packet Dropped

PQ algorithm has the highest throughput value while the FIFO and WFQ have lowest throughput value, as is presented in fig. 32 .

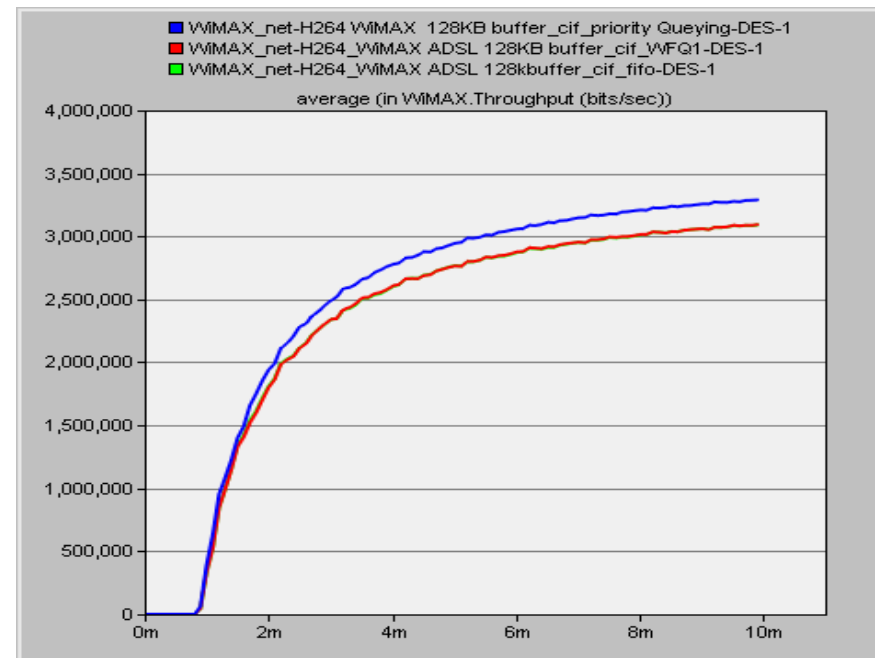

Figure 32: wimax Throughput (bits)

$\mathbf{X}$.

CONCLUSION

The study searched the technical information and behavior or performance of ADSL and WiMAX broadband access technology. This research aims to report performance of ADSL and WiMAX access technology for video streaming applications. Particular conditions and parameter settings are measured in this work. But, there may be differences in the outcomes if the input parameters and conditions are changed. The OPNET designer is used to design and distinguish the behavior of streaming a 10 - minute H.264 to ADSL and WiMAX video subscribers by four performance metrics. Verification scenarios ensured the total design of the work was executed well in the Modeler. The simulation results indicated that while ADSL showed behavior that approximated the ideal values for the performance metrics, first simulation runs displayed significant loss. To develop the total behavior of the network, simulations were examined through different values of buffer size. With more changing, a specification was derived indicating the loss of the packet that was more suitable for the ADSL client station. Performance within the constraints of the well-defined factors. In this research, we calculated the QoS which can be attained by end applications while Integrated Services (IntServ) sub networks are joined together using Differentiated Services network (DiffServ).The proportional network behavior was examined for PQ, FIFO and WFQ with video streaming applications by OPNET simulation tools to accomplish the QoS. In fact, we studied different factors for developing queue technology in more suitable and improved networks. There are different factors probably understated the behavior of the WiMAX access, additionally, video streaming content was exhibited as unicast traffic, however, multicast video traffic may have produced improved behavior. 


\title{
International Advanced Research Journal in Science, Engineering and Technology
}

\author{
Vol. 5, Issue 8, August 2018
}

\section{REFERENCES}

[1] I. Hussain and N. Ahmed, “A Performance Analysis of E-Learning over WiFi-based Long Distance Networks,” vol. 6, no. 4, pp. 85-93, 2016.

[2] J. L. Shah and J. Parvez, "Evaluation of Queuing Algorithms On QoS Sensitive Applications In IPv6 Network," pp. 106-111, 2014.

[3] F. Project, "Comparison of WiMAX and ADSL by Streaming Audio and Video Content," vol. 301136804, pp. 1-38, 2011.

[4] W. Hrudey and L. Trajković, "Streaming Video Content Over IEEE 802 . 16 / WiMAX Broadband Access," Topology, 2008.

[5] K. H. Flayyih, M. Abdul, H. Abbood, and P. Nasser, "H . 264 Video Transmission with High Quality and Low Bitrate over Wireless Network," no. Vcl, pp. 134-145, 2018

[6] L. P. Kondi, A. Luthra, and S. Ci, 4G Wireless Video. 2009.

[7] I. E. G. Richardson, "H.264 and MPEG-4 Video Compression," in Vasa, 2008, pp. 234-256.

[8] P. Abdul, "Error Resilience and Concealment in Multiview Video over Wireless Networks by," no. December, 2014

[9] T. Stockhammer, M. M. Hannuksela, and T. Wiegand, "H . 264 / AVC in Wireless Environments," no. July, pp. 1-28, 2003.

[10] M. S. Al-hawawreh, "Performance Analysis of a WIMAX Network in Different Propagation Models," vol. 15, no. 1, pp. 603-609, 2017.

\section{BIOGRAPHY}

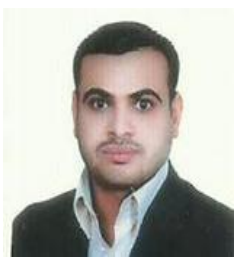

Kadhim Hayyawi Flayyih, born in Al Qadisiyah in 1985 and graduated from the University of Qadisiyah / College of Science at Computer Science Department at 2006- 2007. In 2016 - 2017 I begin the study of a Master in computer science from Iraqi Commission for Computers and Information / Informatics Institute for Postgraduate Studies. 